\title{
Approximating the Minimum Rank of a Graph via Alternating Projection
}

\author{
Franklin H. J. Kenter ${ }^{\mathrm{a}}$ \\ ${ }^{a}$ Department of Computational and Applied Mathematics, Rice University
}

\begin{abstract}
The minimum rank problem asks to find the minimum rank over all matrices with a given pattern associated with a graph. This problem is NP-hard, and there is no known approximation method. Further, this problem has no straightforward convex relaxation. In this article, a numerical algorithm is given to heuristically approximate the minimum rank using alternating projections. The effectiveness of this algorithm is demonstrated by comparing its results to a related parameter: the zero-forcing number. Using these methods, numerical evidence for the minimum rank graph complement conjecture is provided.
\end{abstract}

Keywords: minimum rank of a graph, alternating projections, heuristics, zero-forcing

\section{Introduction}

Seeking the minimum rank of a matrix satisfying given constraints is a well-studied problem with applications in signal processing [11, 24], data analysis 9], topological embeddings [10, as well as economics 22. In general, these problems are NP-hard. However, certain cases, such as the affine minimum rank problem, can be solved exactly or approximately with reliable probabilistic methods and convex optimization [20, 27, 18].

The problem presented here is to minimize the rank over all symmetric matrices with a specified sparsity pattern given by a graph. This sparsity pattern requires certain entries to be zero and others to be nonzero; the requirement that certain entries be nonzero makes the restriction non-affine and nonconvex. Further, these restrictions have no convex relaxation because the zero-matrix lies within the closure of the corresponding set of matrices. Hence, recently developed techniques cannot apply [20, 27, 18.

The biggest obstacle to study of the minimum rank problem for graphs is that not only is there no known effective method to compute the minimum rank of a graph, but also, there are no efficient approximation algorithms either. Our main contribution is the development of an algorithm to approximate the minimum rank for larger graphs. Computationally, the minimum rank problem for graphs is NP-hard 21, and exact computation, in general, has remained elu- sive for anything but very small graphs. Recent results have classified all graphs on $n$ vertices with minimum rank $0,1,2, n-2, n-1$, and $n$ [2. Additionally, the minimum rank for certain families of graphs has been determined exactly [3, 6, 18. However, calculating, or even effectively estimating, the minimum rank of a graph, in general, still remains open.

The minimum rank problem of a graph has a wide range of additional variants and applications. First, the minimum rank of a graph is a relaxation of the Colin de Verdière graph invariant which serves as a strong connection between Schrödinger operators, topological embeddings of graphs, and matrix analysis. In fact, the Colin de Verdière graph invariant gives a spectral characterization for planar graphs [10, 25. In addition, the zero-forcing number of a graph introduced in 2] has been used to bound the minimum rank of a graph from below. We will use the zero-forcing number as a comparison of our analysis; details are given in the next section. While the zeroforcing number has yielded several interesting results in accurately computing the minimum rank for certain families of graphs [21, the zero-forcing number itself is also NP-hard [1. The zero-forcing number is actually adapted from a previous concept known as power domination which serves to optimally place power monitoring units within a power network [4, and its study and application continues to this day [14, 28. Further, recently the study of zero-forcing has been extended to determine the controllability of 
quantum [16, 23, and dynamical systems [19.

We present an iterative algorithm which alternates between minimizing the rank of a matrix and fitting the matrix to the given sparsity pattern. This idea is an extension of the method of alternating projections pioneered by Von Neumann [26] which has since been perfected by others 7,8 and also extended to manifolds [17. Details are given in the next section.

Empirically, this algorithm is effective at accurately computing the minimum rank. We demonstrate the effectiveness of our algorithm by comparing our results to a combinatorial parameter, the zeroforcing number, which bounds the minimum rank from below. Our computational results show that our algorithm is generally close to the theoretical minimum as given by the zero-forcing number. In fact, these results demonstrate that the zero-forcing number is generally a good heuristic approximation for the minimum rank of a graph when the number of vertices is less than 23. This algorithm is efficient for graphs up to 100 vertices. Finally, using our algorithm, we provide numerical evidence for an outstanding conjecture regarding the minimum rank of a graph and its complement. We provide a mathematical foundation that the algorithm does converge when the initial point is sufficiently close to a solution under mild conditions.

This paper is organized as follows. In the next section, we give the preliminaries and definitions. Within Section 3, we present our algorithm and demonstrate its convergence in Section 4. In Section 5. we discuss the computational results of the algorithm and compare them to that of zero-forcing, and in the next section we give numerical evidence to the minimum rank conjecture. Finally, in Section 7, we discuss our conclusions and future work.

\section{Preliminaries}

Let $G=(V, E)$ be a graph with $n$ vertices and $m$ edges. For our purposes, we use simple graphs with no loops or multiedges. The adjacency matrix of $G$, denoted $\mathbf{A}$, is the $n \times n$ symmetric matrix indexed by the vertices where $\mathbf{A}_{i j}=1$ whenever $\{i, j\}$ is an edge and $=0$ otherwise. A weighted adjacency matrix of $G$ is a symmetric $n \times n$ matrix, $\mathbf{W}$, such that for $i \neq j$, $\mathbf{W}_{i j} \neq 0$ whenever $\{i, j\}$ is an edge and $=0$ otherwise, and the diagonal entries may take any values which may be different from one another. Note that in our case, we specifically disallow zero weights but allow negative weights. A matrix that is a weighted adjacency matrix of $G$ is said be a representation of $G$.

Given a graph $G$, the minimum rank problem seeks to minimize $\operatorname{rank}(\mathbf{W})$ over all weighted adjacency matrices of $G$. The optimum value is called the minimum rank of $G$, denoted $m r(G)$. We will use $\operatorname{amr}(G)$ for the output value of our approximation algorithm.

It will be necessary to formulate this problem in terms of manifolds. For our purposes, we assume all manifolds to be smooth and differentiable. We consider the space of $n \times n$ symmetric matrices, $\mathbb{R}_{\text {sym }}^{n \times n}$. For the graph $G$, we define the manifold $\mathcal{S}(G)$ to be the affine subspace in $\mathbb{R}_{\text {sym }}^{n \times n}$ consisting of all representations of $G$. In particular, $\mathcal{S}(G)$ is an affine subspace with select subspaces of lower dimension removed. We will let $\mathcal{S}^{*}(G)$ denote the affine subspace corresponding to the topological closure of $\mathcal{S}(G)$; that is, $\mathcal{S}^{*}(G)$ is the set of all representations of $G$ of all subgraphs of $G$. For $k \leq n$, let $\mathcal{R}_{n}(k)$ be the manifold of all real-symmetric $n \times n$ matrices of rank at most $k$. In which case, the minimum rank problem can be formulated as follows:

$$
\operatorname{mr}(G):=\min k \text { s.t. } \mathcal{S}(G) \cap \mathcal{R}_{n}(k) \neq \emptyset
$$

Additionally, two manifolds $\mathcal{M}, \mathcal{N}$ within $\mathbb{R}_{s y m}^{n \times n}$ intersect transversely at $x \in \mathcal{M} \cap \mathcal{N}$ if the tangent spaces, $T_{\mathcal{M}}, T_{\mathcal{N}}$, have $T_{\mathcal{M}}+T_{\mathcal{N}}=\mathbb{R}_{\text {sym }}^{n \times n}$.

The main tool we use in our algorithm is the method of alternating projections pioneered by von Neumann [26]:

Proposition 1 (von Neumann, see for example [7]). Let $S$ and $T$ be closed subspaces of a Hilbert space, $\mathcal{H}$, and let $\mathbf{P}_{S}$ and $\mathbf{P}_{T}$ be the orthogonal projection operators onto $S$ and $T$ respectively. Then, for any point $h \in \mathcal{H}$,

$\lim _{\ell \rightarrow \infty}\left(\mathbf{P}_{S} \mathbf{P}_{T}\right)^{\ell} h \in S \cap T \quad$ and $\quad \lim _{\ell \rightarrow \infty}\left(\mathbf{P}_{T} \mathbf{P}_{S}\right)^{\ell} h \in S \cap T$

That is, one can find an intersection point of two subspaces by alternating projections onto the two subspaces. This concept has since been perfected by others [7, 8] and also extended to manifolds [17]. In particular, given a metric space $\mathcal{S}$ with distance metric $d$ and two compact sets $S, T \subset \mathcal{S}$, an orthogonal projection from $S$ to $T$ is a function $P: S \rightarrow T$ such that for any $s \in S, P(s)=\arg \min _{t \in T} d(s, t)$. It should be noted that the minimum exists by compactness, and also, an orthogonal projection from one set to another is not necessarily unique.

Let $\mathbf{M}=\mathbf{Q} \Lambda \mathbf{Q}^{T}$ be the real orthonormal diagonalization of a real symmetric matrix $\mathbf{M}$ where $\Lambda$ is 
a diagonal matrix of eigenvalues $\lambda_{1}, \lambda_{2}, \ldots, \lambda_{n}$ with $\left|\lambda_{1}\right| \geq\left|\lambda_{2}\right| \geq \ldots \geq\left|\lambda_{n}\right|$ and $\mathbf{Q}$ is a matrix of (orthogonal) eigenvectors in an order corresponding to the eigenvalues in $\Lambda$. A $k$-rank approximation of $\mathbf{M}$ is $\mathbf{M}_{k}=\mathbf{Q} \Lambda_{k} \mathbf{Q}^{T}$ where $\Lambda_{k}$ is a diagonal matrix with entries $\lambda_{1}, \lambda_{2}, \ldots, \lambda_{k}, 0, \ldots 0$. Note that a $k$-rank approximation is unique (up to a sign on the columns of $\mathbf{Q}$ ) whenever $\mathbf{M}$ has distinct eigenvalues.

We will use $\mathbf{I}$ to denote the identity matrix. Also, for two matrices with the same dimensions, we let $\mathbf{A}$ 。 $\mathbf{M}$ denote the entry-wise (or "Hadamard") product. That is, $(\mathbf{A} \circ \mathbf{M})_{i j}=\mathbf{A}_{i j} \cdot \mathbf{M}_{i j}$.

For matrix norms we will focus upon the Frobenius norm, denoted, $\|\mathbf{M}\|_{F}$, which is given by: $\|\mathbf{M}\|_{F}^{2}=$ $\sum_{i, j} \mathbf{M}_{i, j}^{2}$. Alternatively, $\|\mathbf{M}\|_{F}^{2}=\sum_{k} \sigma_{k}^{2}$ where $\sigma_{k}$ are the singular values of $\mathbf{M}$. For more information, see [15.

In the later part of our paper, we compare our results with the zero-forcing number of a graph. The zero-forcing process originally given in 2] is defined as follows. Start with a set of vertices $S \subset V(G)$ as colored and all other vertices uncolored. If a colored vertex $s$ has all but one of its neighbors colored, then the uncolored neighbor, $t$, changes from uncolored to colored. In which case, we say $s$ forces $t$. This forcing process continues until all of the vertices of the graph become colored or no more vertices can force. If an initial set $S$ eventually forces the entire graph under this forcing process, $S$ is called a forcing set. The zero-forcing number, $Z(G)$, is the size of the smallest forcing set of the graph.

The importance of $Z(G)$ in relation to the minimum rank is the following:

Proposition 2 (AIM Special Work Group [2]).

$$
m r(G) \geq n-Z(G)
$$

Later in our paper, we will utilize the previous proposition in order to evaluate our algorithm for the minimum rank, thereby providing a spread of possible values for $\operatorname{mr}(G)$.

\section{The Algorithm}

The main idea of the algorithm is to alternate between setting $\mathbf{B} \leftarrow \mathbf{B} \circ(\mathbf{A}+\mathbf{I})$ (zeroing out entries) and setting $\mathbf{B} \leftarrow \mathbf{B}_{k}$ (taking the $k$-rank approximation of $B$ ) sufficiently many times. Hence, after each step $\mathbf{B}$ is either a $k$-rank matrix or a representation of $G$ (or a subgraph). Note, that the step $\mathbf{B} \leftarrow \mathbf{B} \circ(\mathbf{A}+\mathbf{I})$ does not turn the matrix $\mathbf{B}$ into a representation of $G$, as it only zeros out entries required to be zero. Rather, it only guarantees that $\mathbf{B}$ is a representation of a subgraph of $G$ (which could be $G$ itself). Hence, if the algorithm converges, it should converge to a matrix that is both rank $k$ and a representation of $G$ or a subgraph of $G$. Afterwards, it is checked if $\mathbf{B} \in \mathcal{S}(G)$ (that is, if $\mathbf{B}$ is a matrix representation of $G$ ). If $\mathbf{B} \in \mathcal{S}(G)$, then $\mathbf{B}$ is a $k$-rank representation of $G$, and hence, the algorithm will lower $k$ in order to find a representation with lower rank. Otherwise, the algorithm with raise $k$ in order to find a representation at all. By using a bisection method with regard to $k$, the algorithm will return the least possible $k$ for which it can find a representation. The main benefit of the algorithm is that not only will it return the minimum rank $k$ it finds, but additionally, it can return a certificate regarding how that rank is achieved numerically.

Data: $\mathbf{A}=$ an adjacency matrix for a graph $G$; $\mathbf{N}=$ a representation of $G ; j_{\max }=$ the maximum number of iterations allowed; $\varepsilon$ $=$ tolerance of precision

Result: $k=$ the approximate minimum rank of $G$; best $\mathbf{B}=$ the representation achieving $k$

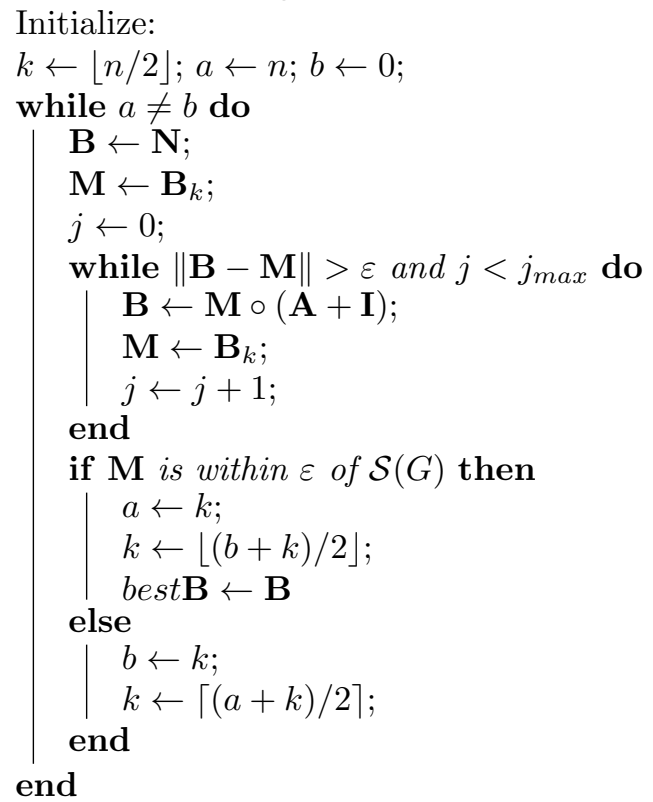

Algorithm 1: Our Algorithm For Estimating the Minimum Rank

We now discuss in more detail the various subroutines including: calculating $\mathbf{B}_{k}$ (the $k$-rank approximation of a matrix); checking if $\mathbf{B} \in \mathcal{S}(G)$ (to see if 
a matrix is in fact a representation of $G$ ), and calculating $\mathbf{B} \circ(\mathbf{A}+\mathbf{I})$.

As for calculating $\mathbf{B}_{k}$, we resort to built-in implementations to calculate the eigenvector- or singularvalue-decomposition. However, we should emphasize that this is the most time-consuming portion of the algorithm. The performance of the algorithm could be greatly improved depending the implementation of this subroutine. Further, in certain cases, such as sparse matrices or when $k$ is small, specific methods might be best.

Determining whether $\mathbf{B} \in \mathcal{S}(G)$ can be tricky, as this checks whether certain entries are non-zero. From a numerical standpoint, this may not be strictly possible by using the results of a numerically convergent algorithm. In many cases, the entries required to be zero may, in fact, be converging to zero but never reach zero. In which case, instead of checking to see if $\mathbf{B} \in \mathcal{S}(G)$, we actually check to see if each entry of $\mathbf{B}$ is within $\varepsilon$ of a matrix in $\mathcal{S}(G)$. For our purposes we use $\varepsilon=10^{-7}$. We should note that from our evaluations, using $\varepsilon=10^{-7}$ is sufficient for graphs with at most 22 vertices whereas $\varepsilon=10^{-4}$ can yield sufficient false results.

\section{Convergence}

In this section, we provide the mathematical framework regarding the algorithm's convergence. In particular, the algorithm obeys the following:

Theorem 1. Given a graph $G$ and $\mathbf{M} \in \mathcal{R}_{n}(k) \cap$ $\mathcal{S}^{*}(G)$, provided $\mathcal{R}_{n}(k), \mathcal{S}^{*}(G)$ intersect transversely at $\mathbf{M}$, then there is an $\varepsilon>0$ such that for any realsymmetric $n \times n$ matrix, $\mathbf{N}$ with $\|\mathbf{M}-\mathbf{N}\|_{F}<\varepsilon$, Algorithm 1 with initial point $\mathbf{N}$ converges to some $\mathbf{M}^{*} \in \mathcal{R}_{n}(k) \cap \mathcal{S}^{*}(G)$.

Our main tool toward proving the theorem above is the following:

Theorem 2 (Alternating Projections on Manifolds, Lewis-Malick, [17] Theorem 13). Let $\mathcal{M}, \mathcal{N}$ be two differentiable manifolds in $\mathbb{R}^{k}$, that intersect transversely at $x \in \mathcal{M} \cap \mathcal{N}$. Let $P_{\mathcal{M}}$ be an orthogonal projection from $\mathcal{M} \rightarrow \mathcal{N}$ and $P_{\mathcal{N}}$ be an orthogonal projection from $\mathcal{N} \rightarrow \mathcal{M}$. Then, there in an $\varepsilon>0$, depending on $x$, such that for any $x_{0} \in \mathcal{N}$ with $\left\|x_{0}-x\right\|<\varepsilon$, the sequence of orthogonal projections defined by

$$
x_{k+1}=P_{\mathcal{N}}\left(P_{\mathcal{M}}\left(x_{k}\right)\right) \text { for } k>0
$$

converges to some $x^{*} \in \mathcal{M} \cap \mathcal{N}$.
Hence, in order to prove Theorem 1 using Theorem 2. we require four elements: $\mathcal{S}^{*}(G)$ is a smooth manifold (Fact 1), $\mathcal{R}_{n}(k)$ is a smooth manifold (Proposition 3), $\mathbf{B} \leftarrow \mathbf{B} \circ(\mathbf{A}+\mathbf{I})$ is an orthogonal projection from $\mathcal{S}^{*}(G)$ to $\mathcal{R}_{n}(k)$ (Lemma 1), and lastly $\mathbf{B} \leftarrow \mathbf{B}_{k}$ is an orthogonal projection from $\mathcal{R}_{n}(k)$ to $\mathcal{S}^{*}(G)$ (Lemma 2).

Fact 1. If $G$ is a graph with $n$ vertices and $m$ edges, the set $\mathcal{S}^{*}(G)$ is an affine subspace in $\mathbb{R}_{\text {sym }}^{n \times n}$ of dimension $n+m$. In particular, it is a smooth manifold.

Proposition 3 (See, for example, [12]). The set $\mathcal{R}_{n}(k)$ is a smooth $(n-k+1)$-dimensional manifold in the space $\mathbb{R}_{s y m}^{n \times n}$

Lemma 1 (See, for example, [15]). Let $\mathbf{M}$ be a symmetric square matrix and let $\mathbf{M}_{k}$ be the best $k$ rank approximation of $\mathbf{M}$ under the Frobenius norm. Then,

$\left|\lambda_{k+1}(\mathbf{M})\right|=\left\|\mathbf{M}-\mathbf{M}_{k}\right\|_{F}=\min _{\mathbf{B}, \operatorname{rank}(\mathbf{B})=k}\|\mathbf{M}-\mathbf{B}\|_{F}$.

Lemma 2. Given a symmetric square matrix $\mathbf{M}$ and an adjacency matrix $\mathbf{A}$ of a graph $G$, then $\mathbf{M} \circ(\mathbf{A}+\mathbf{I})$ is the best approximation of $\mathbf{M}$ within $\mathcal{S}^{*}(G)$ under the Frobenius norm. Specifically, $\mathbf{M} \circ(\mathbf{A}+\mathbf{I})$ obeys:

$$
\|\mathbf{M}-\mathbf{M} \circ(\mathbf{A}+\mathbf{I})\|_{F}=\min _{\mathbf{B}, \mathbf{B} \in \mathcal{S}^{*}(G)}\|\mathbf{M}-\mathbf{B}\|_{F} .
$$

Proof of Theorem 1 , $\mathbf{B} \leftarrow \mathbf{B} \circ(\mathbf{A}+\mathbf{I})$ is an orthogonal projection from $\mathcal{S}^{*}(G)$ to $\mathcal{R}_{n}(k)$ by Lemma 1 , and $\mathbf{B} \leftarrow \mathbf{B}_{k}$ is an orthogonal projection from $\mathcal{R}_{n}(k)$ to $\mathcal{S}^{*}(G)$ by Lemma 2. Hence, applying $\mathcal{M}=\mathcal{R}_{n}(k)$ and $\mathcal{N}=\mathcal{S}^{*}(G)$ completes the proof.

\section{Computational Results}

We now provide several computational results. All these computations were performed in Mathematica on a desktop with $2.93 \mathrm{GHz}$ processor and $16 \mathrm{~GB}$ of memory.

We focus on evaluating $Z(G)$ and our approximation for the minimum rank, $\operatorname{amr}(G)$ over all graphs in the Wolfram database between 9 and 22 vertices, inclusive, covering 2594 graphs. We will use $Z(G)$ to evaluate the effectiveness of $\operatorname{amr}(G)$. We do this because aside from very small graphs and specialized families, the minimum rank for graphs is, in general, 
unknown and difficult to compute [1]. Additionally, to our knowledge, there are no reasonable convex relaxations of the minimum rank problem, so our results cannot be compared to general rank minimization methods such as [20, 27, [18. Hence, as a basis for comparison, we use Proposition 2 $n-Z(G) \leq$ $\operatorname{mr}(G)$, and equality holds for all graphs with 7 or fewer vertices [2]. Hence, if our computations approximate the minimum rank with $\operatorname{amr}(G)=n-Z(G)$, then our approximation for the minimum rank is, in fact, very likely exact.

We compute $\operatorname{amr}(G)$, over all graphs in the Wolfram database between 9 and 22 vertices, inclusive, covering 2594 graphs. For these calculations, we consider 21 initial conditions: $\mathbf{A}-\lambda \mathbf{I}$ for $\lambda=$ $0,0.1, \ldots, 1.9,2$ where $\mathbf{A}$ is the adjacency matrix. The choice of these initial conditions is based upon an initial test that showed that the optimal value for the minimum rank is often achieved when the initial matrix is $\mathbf{A}-\lambda \mathbf{I}$ for $\lambda \in[0,2]$. We then use the best value among the 21 outputs to determine $\operatorname{amr}(G)$. This computation takes only a matter of hours even though it considers 21 different initial matrices for each of the approximately 2600 graphs.

For $Z(G)$, we apply the greedy approach to find an upper bound for $Z(G)$, then apply a brute-force approach by trying all applicable subsets of a smaller sizes, decreasing the size as necessary until no forcing set is found. Since this is a brute force approach, the run-time of the algorithm grows exponentially, and we find it infeasible for graphs with more than 22 vertices.

It is important to note that since our algorithm typically returns a numerical certificate, for most practical purposes, our algorithm will return an upper bound. However, due to the numerical nature of the algorithm, it is potentially possible for $\operatorname{amr}(G)$ to be less than $m r(G)$. For example, the algorithm may interpret small singular values (i.e. below $\varepsilon$ which we take to be $10^{-7}$ ) to be exactly 0 , when they converge to a nonzero value. None the less, in all cases up to 23 vertices, the computed value, $\operatorname{amr}(G)$, was never less than $n-Z(G)$, a lower bound of $m r(G)$, suggesting that $\operatorname{amr}(G)$ is rarely less than $\operatorname{mr}(G)$.

Our results can be found in Figure 1 where we compute the quantity $\operatorname{amr}(G)-(n-Z(G))$ where $\operatorname{amr}(G)$ is our approximation for the minimum rank. This plot shows that the average value for $\operatorname{amr}(G)-(n-$ $Z(G))$ on all named graphs with $n$ vertices $(n \leq 22$, with the exception of $n=20)$ is less than 0.6. By Proposition 2, $n-Z(G) \leq m r(G)$. Hence, unless numerical errors cause $\operatorname{amr}(G)<m r(G)$, we can conclude that $\operatorname{mr}(G)=\operatorname{amr}(G)$ for at least $40 \%$ of small graphs. Further, one should note there are graphs for which the minimum rank is strictly greater than $n-Z(G)$. Hence, the performance of the algorithm may be better than the gap shown in Figure 1 Hence, experimentally, our algorithm appears be be a good heuristic for the zero-forcing number as well for graphs with at most 22 vertices.

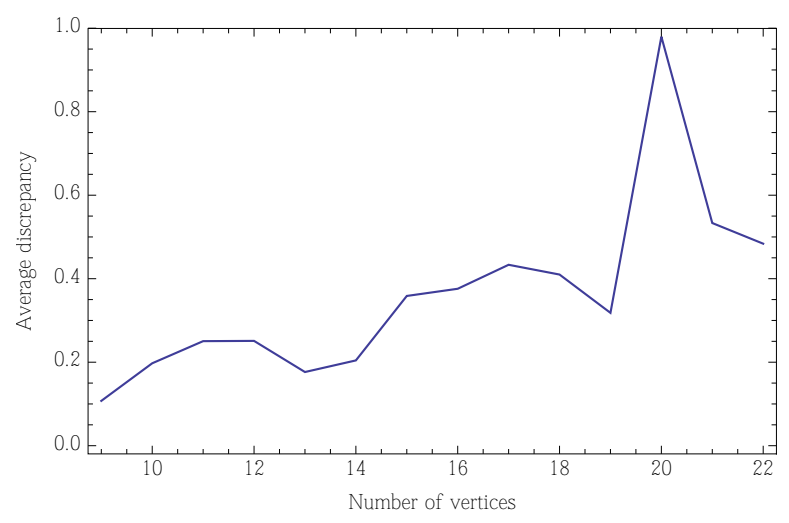

Figure 1: A plot of the average value of $\operatorname{amr}(G)-(n-Z(G))$ over graphs from the Wolfram database

In order to determine the limitations of our algorithm, we apply the algorithm on Erdös-Reyni random graphs, $G(n, p)$. Specifically, we generate random graphs on $n$ vertices where each potential edge is realized with probability $p$. The specific case of $G(n, 1 / 2)$ has been studied in the context of the minimum rank problem. In fact, $0.14 n<\mathbb{E}(m r(G(n, 1 / 2)))<0.5 n+\sqrt{n \ln \ln n}$ where $\mathbb{E}(\operatorname{mr}(G(n, 1 / 2)))$ is the expected value of the minimum rank [13]. The authors believe that upper bound is asymptotically tight 13 . We apply our algorithm to random realizations $G(n, 1 / 2)$ to increas-

\begin{tabular}{l||c||c|c}
$\mathrm{n}$ & $a m r(G)-(n-Z(G))($ mean $)$ & $a m r(G)(\mathrm{sec})$ & $Z(G)$ time $(\mathrm{sec})$ \\
\hline 9 & 0.107383 & 0.575857 & 0.045272 \\
10 & 0.197425 & 0.599819 & 0.050206 \\
11 & 0.250432 & 0.737648 & 0.106382 \\
12 & 0.251046 & 0.943793 & 0.230569 \\
13 & 0.176471 & 0.944322 & 0.324729 \\
14 & 0.204283 & 1.538733 & 1.170821 \\
15 & 0.358696 & 1.420584 & 2.060554 \\
16 & 0.375887 & 1.864205 & 3.446570 \\
17 & 0.433333 & 2.617768 & 6.909289 \\
18 & 0.409938 & 3.643548 & 15.787273 \\
19 & 0.318182 & 3.590746 & 22.881866 \\
20 & 0.979798 & 3.957805 & 71.958347 \\
21 & 0.533333 & 4.902006 & 187.263572 \\
22 & 0.483871 & 6.073192 & 324.366621 \\
\hline
\end{tabular}

Figure 2: A table of the average value of $\operatorname{amr}(G)-(n-Z(G))$ and the run-times for $\operatorname{amr}(G)$ and $Z(G)$. Since $\operatorname{amr}(G)-(n-$ $Z(G))$ is close to 0 for most cases, $m r(G) \leq n-Z(G)$, and for practical purposes $\operatorname{amr}(G) \geq m r(G), \operatorname{amr}(G)=m r(G)$ for a large proportion of small graphs. 


\begin{tabular}{l||c||c||c||}
$\mathrm{n}$ & trials & mean $a m r(G(n, 1 / 2))$ & $a m r(G)$ time $(\mathrm{s})$ \\
\hline 50 & 40 & 22.1395 & 39.300171 \\
60 & 40 & 25.7674 & 64.752044 \\
70 & 40 & 30.8605 & 105.935827 \\
80 & 40 & 35.6047 & 156.254314 \\
90 & 40 & 41.9302 & 234.622186 \\
100 & 40 & 48.4545 & 335.205289 \\
200 & 1 & 116 & 3872.132177 \\
300 & 1 & 179 & 19152.084420 \\
400 & 1 & 201 & 28224.679512 \\
\hline
\end{tabular}

Figure 3: The average values of $\operatorname{amr}(G(n, 1 / 2))$ for increasing values of $n$. The approximations for minimum rank are in line with the theoretical results in [13].

ing values of $n$. Since the minimum rank of the graph is large relative to $n$, the algorithm will be recursively applying dense eigenvalue-compositions. None the less, the algorithm is effective up to roughly 100 vertices running in less than 6 minutes. The runtime of the algorithm appears to grow significantly thereafter, timing out above 400 vertices. In most all cases the mean value of the calculated minimum rank $\operatorname{amr}(G)$ is approximately $\frac{1}{2} n$ which is close to the theoretical threshold above predicted to be accurate. These results, including the corresponding run-times, are summarized in Figure 3.

\section{The Graph Complement Conjecture}

Using the algorithm, we can provide numerical evidence for the Graph Complement Conjecture:

Conjecture 1 (Graph Complement Conjecture, weak form [5]). Let $G$ be an undirected graph on $n$ vertices. Then, for some universal constant $\alpha<2$,

$$
m r(G)+m r(\bar{G}) \leq \alpha n
$$

where $\bar{G}$ denotes the graph complement.

The complement of $G, \bar{G}$, is the graph where $\{i, j\}$ is an edge of $\bar{G}$ if and only if $\{i, j\}$ is not an edge in $G$. In fact, a stronger form conjectures the $\alpha n$ can be replaced by $n+2$; this would be sharp [5]. The strong form of the conjecture has only been settled for specialized families of graphs and also when $\operatorname{mr}(G) \leq 4$ [5. None the less, the other variations of the conjecture remain open. Until now, no numerical evidence has been provided for the Graph Complement Conjecture.

We apply our algorithm in order to numerically test our conjecture on named graphs in the Wolfram database of at most 50 vertices, consisting of over 6300 graphs. Unlike previously, we only use 9

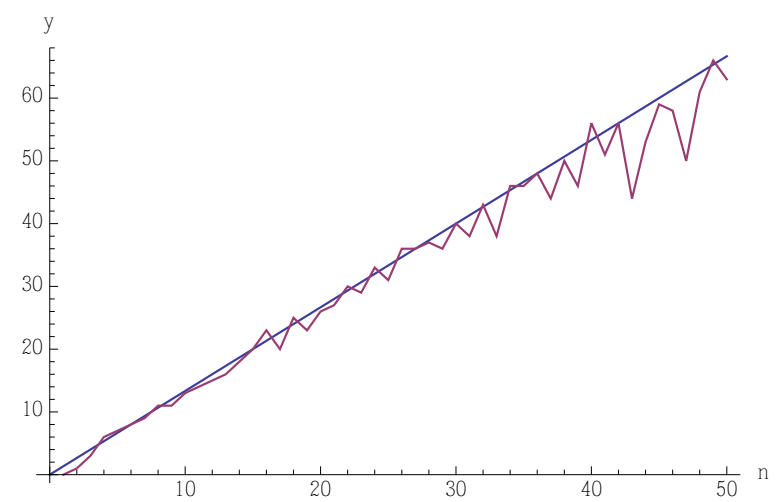

Figure 4: A plot of the maximum value of $\operatorname{amr}(G)+a m r(\bar{G})$ over all graphs in the database on $n$ vertices against the line $y=\frac{4}{3} n$

initial matrices for a faster run time: $\mathbf{A}-\lambda \mathbf{I}$ for $\lambda=0,0.25, \ldots, 2$. However, note that in this computation, we must also perform the algorithm on $\bar{G}$ in addition to $G$.

The results of this algorithm can be seen in Figure 4 where we plot the maximum value of $\operatorname{amr}(G)+$ $\operatorname{amr}(\bar{G})$ over all graphs with $n$ vertices. In fact, this computation strongly suggests the weak form of the graph complement conjecture is most probably true as all graphs in this calculation have $(\operatorname{amr}(G)+$ $\operatorname{amr}(\bar{G})) / n<1.44$

\section{Conclusion and Future Work}

We remark there are several interesting avenues for future work. First, recent work has been done extending Newton's Method to manifolds. Typically, Newton's method runs in quadratic time whereas alternating projections depends on the angle of intersection between the two sets [17. Hence, adapting our methods to work with Newton's method should produce a faster and more effective algorithm. In addition, since the minimum rank of a graph is related to many other graph-theoretical parameters such as zero-forcing, power domination, and the Colin de Verdière invariant, the technique presented here may prove helpful toward generating approximations and heuristics for these other applications.

\section{Acknowledgments}

This research was partially supported by NSF CMMI-1300477 and CMMI-1404864. 
[1] AazAmi, A. Hardness results and approximation algorithms for some problems on graphs. $\mathrm{PhD}$ thesis, University of Waterloo, 2008.

[2] "AIM Special Work Group". Zero forcing sets and the minimum rank of graphs. Linear Algebra and its Applications 428, 7 (2008), 16281648.

[3] Almodovar, E., Deloss, L., Hogben, L., Hogenson, K., Murphy, K., Peters, T., And Ramírez, C. A. Minimum rank, maximum nullity and zero forcing number for selected graph families. Involve (2010), 371-392.

[4] Baldwin, T., Mili, L., Boisen Jr, M., And ADAPA, R. Power system observability with minimal phasor measurement placement. Power Systems, IEEE Transactions on 8, 2 (1993), 707-715.

[5] Barioli, F., Barrett, W., Fallat, S. M., Tracy Hall, H., Hogben, L., and van DeR Holst, H. On the graph complement conjecture for minimum rank. Linear Algebra and its Applications 436, 12 (2012), 4373-4391.

[6] Barioli, F., Fallat, S., and Hogben, L. Computation of minimal rank and path cover number for certain graphs. Linear Algebra and its Applications 392 (2004), 289-303.

[7] Bauschke, H., And Borwein, J. M. On the convergence of von Neumann's alternating projection algorithm for two sets. Set-Valued Analysis 1, 2 (1993), 185-212.

[8] Boyle, J. P., And Dykstra, R. L. A method for finding projections onto the intersection of convex sets in Hilbert spaces. In Advances in order restricted statistical inference. Springer, 1986 , pp. $28-47$.

[9] Cai, J.-F., Candès, E. J., And Shen, Z. A singular value thresholding algorithm for matrix completion. SIAM Journal on Optimization 20, 4 (2010), 1956-1982.

[10] Colin de Verdière, Y. Sur un nouvel invariant des graphes et un critère de planarité. Journal of Combinatorial Theory, Series B 50, 1 (1990), 11-21.

[11] El Gamal, H., and Hammons JR., A. R. A new approach to layered space-time coding and signal processing. Information Theory, IEEE Transactions on 47, 6 (2001), 2321-2334.

[12] Guillemin, V., And Pollack, A. Differential topology, vol. 370. American Mathematical Soc., 2010.

[13] Hall, H. T., Hogben, L., Martin, R., And SHADER, B. Expected values of parameters associated with the minimum rank of a graph. Linear Algebra and its Applications 433, 1 (2010), 101-117.

[14] Haynes, T. W., Hedetniemi, S. M., HedetNiemi, S. T., And Henning, M. A. Domination in graphs applied to electric power networks. SIAM Journal on Discrete Mathematics 15, 4 (2002), 519-529.

[15] Hogben, L. Handbook of Linear Algebra. CRC Press, 2006.

[16] Hogben, L., Burgarth, D., D’Alessandro, D., Severini, S., And Young, M. Zero forcing, linear and quantum controllability for systems evolving on networks.

[17] Lewis, A. S., ANd Malick, J. Alternating projections on manifolds. Mathematics of Operations Research 33, 1 (2008), 216-234.

[18] LiU, Z., And VAndenberghe, L. Interior-point method for nuclear norm approximation with application to system identification. SIAM Journal on Matrix Analysis and Applications 31, 3 (2009), 1235-1256.

[19] Monshizadeh, N., Zhang, S., and CamliBEL, M. K. Zero forcing sets and controllability of dynamical systems defined on graphs.

[20] Recht, B., Fazel, M., and Parrilo, P. A. Guaranteed minimum-rank solutions of linear matrix equations via nuclear norm minimization. SIAM review 52, 3 (2010), 471-501.

[21] Renegar, J. On the computational complexity and geometry of the first-order theory of the reals. part iii: quantifier elimination. Journal of Symbolic Computation 13, 3 (1992), 329-352.

[22] Samuelson, P. Foundations of economic analysis. Cambridge, Massachusetts (1948).

[23] Severini, S. Nondiscriminatory propagation on trees. Journal of Physics A: Mathematical and Theoretical 41, 48 (2008), 482002. 
[24] Tarokh, V., Jafarkhani, H., and CalderBANK, A. R. Space-time block codes from orthogonal designs. Information Theory, IEEE Transactions on 45, 5 (1999), 1456-1467.

[25] Van Der Holst, H., Lovász, L., ANd SchriJVER, A. The Colin de Verdière graph parameter. Graph Theory and Computational Biology (Balatonlelle, 1996) (1999), 29-85.

[26] Von Neumann, J. On rings of operators reduction theory. Annals of Mathematics (1949), 401-485.

[27] Wright, J., Ganesh, A., Rao, S., Peng, Y., AND MA, Y. Robust principal component analysis: Exact recovery of corrupted low-rank matrices via convex optimization. In Advances in neural information processing systems (2009), pp. 2080-2088.

[28] Zhao, M., Kang, L., and Chang, G. J. Power domination in graphs. Discrete mathematics 306, 15 (2006), 1812-1816. 